\title{
The Relationship Between Preference Strength and Relative Frequency of Choice
}

\author{
Dan Zakay \\ Tel Aviv University
}

Probability of choice is the primary method of measurement suggested by stochastic models of choice for validating decision-making models. It can be measured by relative frequency of choice (RF) or by preference strength (PS). It is suggested in this research that the relationship between PS values and RF values is not a linear but a cubic function, in the lower section of which RF is zero. In the middle, RF is monotonically related to PS, and in the upper section, RF is always 1.0. Twenty subjects performed decision tasks resulting in 400 pairs of PS and RF values. The hypothesized relationship between RF and PS was supported by the analyses. The methodological and theoretical implications are discussed.

Probability of choice is the primary method of measurement suggested by stochastic models of choice, and is usually estimated by relative frequency of choice (RF). However, other measures and interpretations, such as confidence judgments (Tversky, 1972) and preference strength (Suydam \& Myers, 1962), might also be adopted. These last two methods are applicable when the single presentation method is employed, i.e., the presentation of each stimulus only once. Suydam (1965) claimed that under repeated presentation, choice is, in part, a function of sequential effects, feedback, and set. This idea is supported by many other researchers (Anderson, 1959; Anderson \& Whaler,

APPLIED PSYCHOLOGICAL MEASUREMENT

Vol. 8, No. 2, Spring 1984, pp. 207-211

(1) Copyright 1984 Applied Psychological Measurement Inc. 0146-6216/84/020207-05\$1.50
1960; Jarvic, 1951; Myers \& Fort, 1963). Coombs and Komorita (1958) found that the choice behavior of subjects who were repeatedly offered a number of options differed from that of subjects who were offered each of these options only once. Sequential effects as well as the effects of negative motivation caused by boredom can be eliminated effectively by the method of single presentation. Suydam and Myers (1962) used this method, and their subjects rated their strength of choice on a 9point scale. Their findings confirmed previous results (Anderson \& Whaler, 1960) that suggested that rating scales can provide a more sensitive measure of preference than choice alone.

According to several choice models (e.g., Ajzen \& Fishbein, 1969; Wilkie \& Pessemier, 1973), the outcome of the internal decision process is expressed as some kind of belief or attitude toward each alternative. It is argued here that such an outcome is directly expressed as a feeling of preference strength (PS) toward each alternative in a decision situation. On the other hand, although the overt choice which determines RF is a function of PS, RF is less sensitive than PS. Hence, the relationship between choice probabilities as measured by PS and RF is not expected to be linear. Figure 1 presents the hypothesized relationship between PS and RF.

As can be seen, when PS is greater than a certain value $\left(\mathrm{PS}_{2}\right)$, RF will always tend to be 1.0 . When $\mathrm{PS}$ is smaller than a certain value $\left(\mathrm{PS}_{1}\right), \mathrm{RF}$ will 
Figure 1

The Hypothesized Relationship Between PS and RF

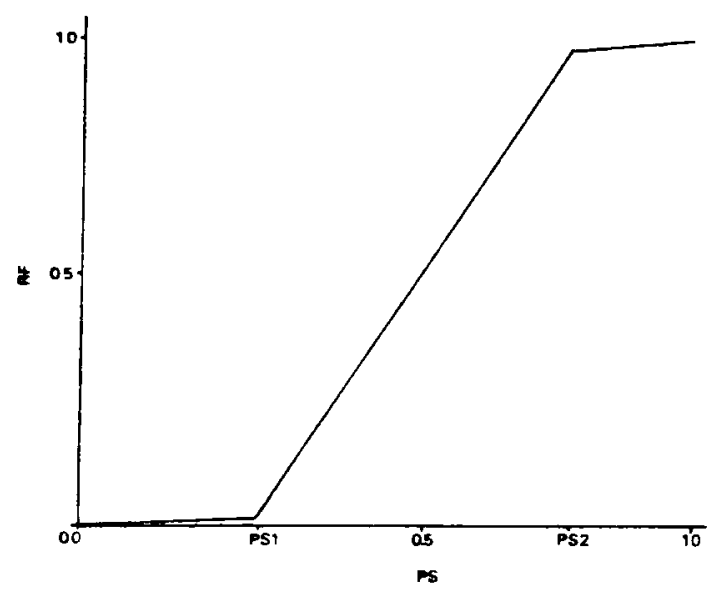

tend to be zero, where $\mathrm{PS}_{1}$ and $\mathrm{PS}_{2}$ are idiosyncratic measures. In the region where $\mathrm{PS}_{1}<\mathrm{PS}<\mathrm{PS}_{2}$, the relationship with RF will be monotonic, and when PS is around .5, RF will tend to be similar. The reason for this is that while it is possible to experience various degrees of PS, the actual choice in each choice is dichotomous, i.e., choosing or not choosing. Hence, if PS, is large enough in each choice, alternative $j$ will be chosen and its RF of choice across repeated choices will be 1.0. If PS, is small enough in each choice, alternative $j$ will not be chosen and its RF of choice across repeated choices will be zero. If $\mathrm{PS}_{J}$ is between $\mathrm{PS}_{1}$ and $\mathrm{PS}_{2}$, a feeling of doubt and conflict will result, and $\mathrm{PS}_{j}$ will be chosen with a proportion of times reflecting more or less PS. The aim of the present study was to test this hypothesized relationship between choice probabilities when measured by RF and PS.

\section{Method}

\section{Subjects}

Twenty students in an introductory psychology course participated in the experiment in partial fulfillment of course requirements.

\section{Decision tasks}

Twenty binary choice situations were composed for the purpose of this experiment. Each was composed of two alternatives that were descriptions of applicants to the Department of Mathematics. The decision task was to choose the most suitable candidate. Information was given on intelligence, motivation, and matriculation exams. The subjects were told the ranges of the scales on which the scores were given. (The intelligence scale ranged from 80 to 150 ; the motivation and matriculation scales ranged from zero to 100.) For example, in one choice situation, alternative 1 was 130 in intelligence, 90 in motivation, and 90 in matriculation; whereas alternative 2 was 120 in intelligence, 90 in motivation, and 95 in matriculation. These choice situations were designed so that the relations between the two alternatives in each situation covered the entire range from strong dominance of one alternative over the other to equality of both alternatives.

A second type of binary choice situation was designed. Each one of these was composed of two alternatives in a roulette gambling situation. Information was provided regarding the probability of red or black winning, as well as the amount of money (in Israeli currency) to be won in each color.

\section{Procedure}

Each subject was tested individually and received the 20 applicant choice situations 10 times. In other words, each subject made 200 choices. The order of choices was random and differed from subject to subject, although two identical choices never appeared consecutively. Ten times during the experiment various roulette choice situations were presented in order to make the task more interesting and to mask previous applicant choices. However, the roulette choices were not used in the statistical analysis since each subject made 10 different choices of that type-each choice only once.

At the beginning of the experiment each subject received two examples of both choice paradigms and the experimental tasks were demonstrated to him/her. The subject was asked to choose one of 
the two alternatives in each situation and to relate to each situation as if it were the first time the situation was being faced. In addition, on 2 out of 10 times in each applicant-choice situation, the subject was asked initially to rate his/her preference strength towards each alternative before reporting his/her actual choice. ${ }^{1}$ This was done by means of a numeric scale ranging from 0 to 100 , where 0 stood for "This alternative is not preferred at all, in comparison to the other one," and 100 stood for "This alternative is absolutely preferred to the other one." One of these PS ratings was done on a choice situation randomly chosen from the first five appearances of each applicant-choice situation, and the second PS rating was from the second five appearances. The experiment lasted about $90 \mathrm{~min}$ utes.

\section{Analyses and Results}

As mentioned earlier, each subject made 20 applicant choices, each choice ten times over. Thus, with regard to each choice, the RF of each alternative (out of 10) was calculated. In addition, with regard to 2 out of 10 choices, each subject reported his/her $\mathrm{PS}, \mathrm{PS}_{1}$ and $\mathrm{PS}_{2}$. The reliability of this measure was found to be very high $(r=.91, d f$ $=18, p<.01$ ). Hence, for each choice, the PS was computed as the average of $\mathrm{PS}_{1}$ and $\mathrm{PS}_{2}$. In this way, 400 results were obtained, with each including an RF and a PS. With respect to each applicant-choice situation, one alternative was randomly chosen ahead of time, and its RF and PS were then measured and analyzed.

The PS scale, between 0 and 100, was divided into 20 equal intervals of five points each. For each such interval, the average RF was computed $\left(\mathrm{RF}_{A}\right)$ as well as the average PS (PS ${ }_{A}$ ) across all results falling into that interval. The relationship between $\mathrm{PS}_{A}$ and $\mathrm{RF}_{A}$ was plotted, and is presented in Figure 2 .

\footnotetext{
${ }^{1}$ Direct measures of PS and subjective probability are common in the frame of Bayesian research and integration theory (e.g., Anderson, 1974a, 1974b; Beach \& Wise, 1969; Lee, 1971; Peterson \& Miller, 1965).
}

As can be seen clearly from Figure 2, the relationship between $\mathrm{PS}_{A}$ and $\mathrm{RF}_{\mathrm{A}}$ is nonlinear. The curve that represents this relationship is composed of at least three sections. In the lower and upper sections there is no relationship between PS and RF, mainly because in these sections the variance of RF is small. In the middle section, however, the relationship appears to be linear.

For the sake of the next analysis, the PS scale was arbitrarily divided into three sections: $S_{1}, S_{2}$, and $S_{3}$, so that $0 \leqslant S_{1} \leqslant .30 ; .30<S_{2} \leqslant .70$; and $.70<S_{3} \leqslant 1.00$. This arbitrary division was done on the basis of the assumption that if the hypothesized relationship is valid, then in $S_{1}$, PS values should be higher than the corresponding RF values. In $S_{2}$, they should be similar, and in $S_{3}$, RF values should be higher than PS values, regardless of the exactness of the cutting points.

The distribution of the 400 results across the three sections was: 127 in $S_{1}, 79$ in $S_{2}$, and 194 in $S_{3}$. Trend analysis was performed on $\mathrm{RF}_{A}$ for each one of the sections and on the entire distribution, using the 5-point intervals as groups in the analysis. In $S_{1}$ and in $S_{3}$ no significant trend was found. In $\mathrm{S}_{2}$ a significant linear trend was found $(F(1,8)=$ $36.11, p<.01)$. The overall trend analysis showed a significant linear trend $(F(1,2)=65.18, p<$ $.01)$, and a significant cubic trend $(F(1,20)=$ $5.16, p<.05)$. In addition, a Pearson correlation was calculated between PS values and RF values for each section using all pairs of values falling into each section. In $S_{1}, r$ was .06 , in $S_{3}, r$ was $.21(d f=192, p<.05)$, and in $S_{2}, r$ was $.62(d f$ $=77, p<.01)$. There was no point in computing the correlation for the entire distribution because of the cubic component found in the trend analysis.

An ANOVA was performed on the three sections comparing $\mathrm{PS}_{A}$ and $\mathrm{RF}_{A}$, in a repeated measures design. A significant main effect among the sections was found but this was trivial. However, a significant interaction between the type of measurement (i.e., PS or RF) and the sections was found $(F(1,38)=6.83, p<.05)$. The means of PS and RF were 15.6 and 5.8 in $S_{1} ; 49.9$ and 47.4 in $S_{2}$; and 86.7 and 92.9 in $S_{3}$, respectively.

The curve showing $\mathrm{RF}_{A}$ values against $\mathrm{PS}_{A}$ values was plotted for each subject individually. Two 
Figure 2

The Relationship Between $\mathrm{PS}_{A}$ and $\mathrm{RF}_{A}$ in the Applicant Choices

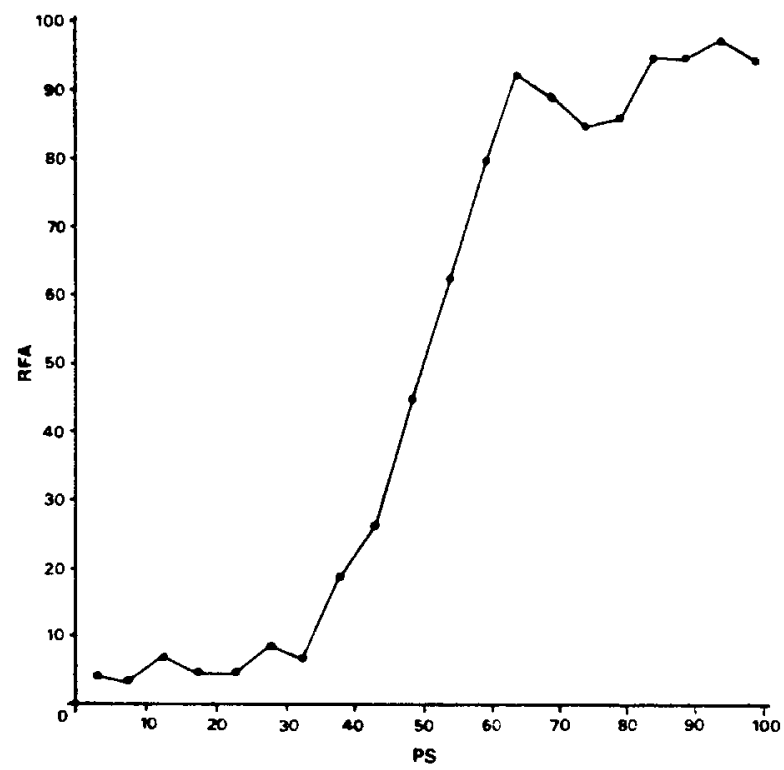

distinct groups emerged: One $(n=15)$ in which all individual curves resembled the hypothesized relationship to a large degree, and a second group $(n=5)$ in which individual curves showed marked deviations from it. The number of transitivity violations across the 20 binary applicant choices done by each subject was counted. The mean was 3.47 in the first group and 5.60 in the second, and the difference was found to be significant $(t=1.91$; $d f=18 ; p<.05$, one-tailed test $)$. This last finding is in support of the possibility that the deviations in the second group reflected a specific state of mind at the moment of choice, e.g., boredom, fatigue, or resistance, leading to random choices or to a choice contrary to feeling.

\section{Discussion}

The results obtained in this research support the existence of the hypothesized relationship between PS and RF, and indicate that it is not linear but composed of a combination of linear and cubic trends. The curve representing the relationship can be divided into three sections, according to the original hypothesis. The cutting points between the three sections seem to be idiosyncratic. Defining $\mathrm{PS}_{1}$ as the last point at which RF equals zero, and $\mathrm{PS}_{2}$ as the first point after which all RF values equal 1.0 , and applying these definitions to the individual curves obtained, the result is $\mathrm{PS}_{1}$ ranging from 20 to 54 , and $\mathrm{PS}_{2}$ ranging from 65 to 83 . Accordingly, there is no sense in calculating any cutting points based on group data. That implies, in turn, that single PS judgments of single individuals are uninformative, because a set of such judgments is required for each individual to norm them. This is a constraint on the use of PS judgments as predictors of corresponding RF values.

However, the type of relationship found in this research holds an important theoretical implication for the psychological measurement of choice probability. It seems that PS is a more sensitive measure than RF. It is assumed that PS, rather than RF, is more directly related to the behavioral intent or the attitude formed in the decision process toward each alternative. In this sense, PS is a reflection of attitude while RF is a reflection of overt behavior, and the gap between PS and RF can be considered 
to be a specific case of the general attitude behavior gap (Kreitler \& Kreitler, 1976). This view is supported by Einhorn and Hogarth (1981), who make a distinction between judgment and choice and say that, although the two are related, it is more accurate to see judgment as an aid to choice.

According to this line of reasoning, PS is connected to judgment while RF is a product of choice. However, in view of the constraint mentioned earlier, appropriate methods for PS measurements that will enable it to replace RF as an accurate measurement of choice probability should be developed. The present research should be considered as a first step only towards more fully understanding this important issue. In such future research, conjoint measurement models as well as item response theory could be used as a framework for investigating the PS-RF relationship.

\section{References}

Ajzen, I., \& Fishbein, M. (1969). The prediction of behavioral intention in a choice situation. Journal of Experimental Social Psychology, 5, 400-416.

Anderson, N. H. (1959). Effect of a first order conditional probability in a two choice learning situation. Journal of Experimental Psychology, 59, 79-93.

Anderson, N. H. (1974a). Algebraic models for information integration. (Technical Report No. 45). San Diego: University of California, Center for Human Information Processing.

Anderson, N. H. (1974b). Methods for studying information integration. (Technical Report No. 46). San Diego: University of California, Center for Human Information Processing.

Anderson, N. H., \& Whaler, R. E. (1960). Likelihood judgment and sequential effects in a two choice probability learning situation. Journal of Experimental Psychology, 60, 111-120.

Beach, A. L., \& Wise, J. A. (1969). Subjective probability and decision strategy. Journal of Experimental Psychology, 79, 133-138.
Coombs, C. H., \& Komorita, S. S. (1958). Measuring utility of money through decisions. American Journal of Psychology, 71, 383-389.

Einhorn, H. J., \& Hogarth, R. M. (1981). Behavioral decision theory: Processes of judgment and choice. Annual Review of Psychology, 32, 53-88.

Jarvic, M. E. (1951). Probability learning and a negative recency effect in the serial anticipation of alternative symbols. Journal of Experimental Psychology, 41, 291-297.

Kreitler, H., \& Kreitler, S. (1976). Cognitive orientation and behavior. New York: Springer.

Lee, W. (1971). The effects of expected value difference and expected regret ratio on preference strength. American Journal of Psychology, 84, 194-204.

Myers, J., \& Fort, J. G. (1963). A sequential analysis of gambling behavior. Journal of General Psychology, 69, 299-309.

Peterson, C. R., \& Miller, A. J. (1965). Sensitivity of subjective probability revision. Journal of Experimental Psychology, 70, 117-121.

Sudyam, M. M. (1965). Effects of cost and gain ratios and probability of outcome on ratings of alternative choices. Journal of Mathematical Psychology, 2, 171179.

Suydam, M. M., \& Myers, J. L. (1962). Some parameters of risk-taking behavior. Psychological Reports, $10,559-562$.

Tversky, A. (1972). Elimination by aspects: A theory of choice. Psychological Review, 76, 31-48.

Wilkie, L. W., \& Pessemier, E. A. (1973). Issues in marketing's use of multi-attribute attitude models. Journal of Marketing Research, 10, 428-441.

\section{Acknowledgment}

The author thanks Efraim Bichler for his assistance in conducting this research.

\section{Author's Address}

Send requests for reprints or further information to Dan Zakay, Department of Psychology, Tel Aviv University, Ramat Aviv, 69978, Israel. 